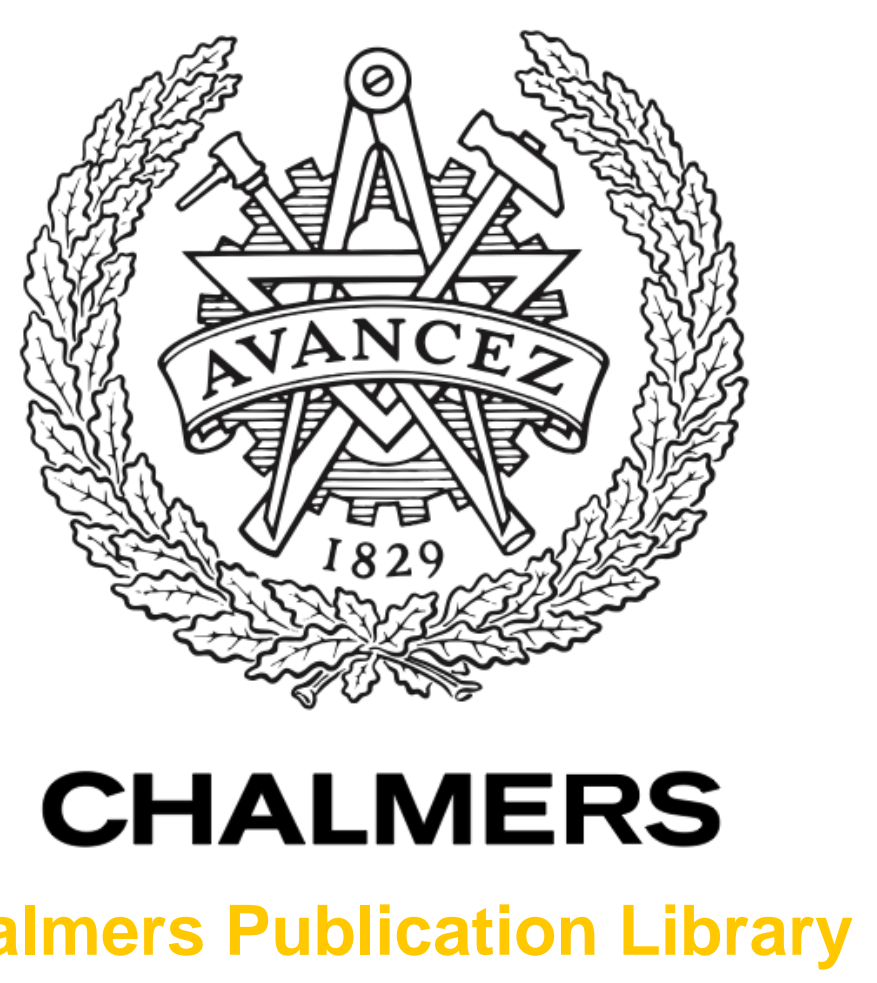

Chalmers Publication Library

\title{
Nonlinear fiber capacity
}

This document has been downloaded from Chalmers Publication Library (CPL). It is the author's version of a work that was accepted for publication in:

Proc. European Conference on Optical Communication (ECOC) 2013, London, U.K. [Invited]

Citation for the published paper:

Agrell, E. (2013) "Nonlinear fiber capacity". Proc. European Conference on Optical Communication (ECOC) 2013, London, U.K. [Invited]

Downloaded from: http://publications.lib.chalmers.se/publication/187246

Notice: Changes introduced as a result of publishing processes such as copy-editing and formatting may not be reflected in this document. For a definitive version of this work, please refer to the published source. Please note that access to the published version might require a subscription. 


\title{
Nonlinear Fiber Capacity
}

\author{
Erik Agrell \\ Department of Signals and Systems, Chalmers University of Technology, Gothenburg, Sweden. \\ $\bowtie$ agrell@chalmers.se
}

Abstract In this semi-tutorial presentation, we review fundamental information theory for links with and without memory, in the linear and nonlinear regimes. A comparison between channel models with long (but finite) memory and infinite memory yields an unexpected result.

\section{Introduction}

Shannon, the father of information theory, proved that for a given channel, it is possible to achieve an arbitrarily small error probability, if the transmission rate in bits per symbol is small enough. A rate for which virtually error-free transmission is possible is called an achievable rate and the supremum over all achievable rates for a given channel, represented as a statistical relation between its input $X$ and output $Y$, is defined as the channel capacity [1], [2, p. 195]. A capacity-approaching transmission scheme operates in general by grouping the data to be transmitted into blocks, encoding each block into a sequence of information symbols, transmitting this sequence over the channel, and decoding it in the receiver. A long block length is essential to obtain an arbitrarily small error probability, even for memoryless channels, and for channels with memory, the block length should typically be much larger than the channel memory.

\section{The GN model and its capacity}

For coherent long-haul fiber-optical links without dispersion compensation, the most popular discretetime channel models represents the nonlinear interference (NLI) as Gaussian noise (GN), whose statistics depend on the transmitted signal power [3-6]. Thus, the complex single-channel output $Y_{n}$ is modeled as

$$
Y_{n}=X_{n}+Z_{n}
$$

where $X_{n}$ is the complex channel input and $Z_{n}$ is a complex, white, Gaussian random sequence, independent of $X_{n}$. Although $Z_{n}$ is independent of the actual transmitted process $X_{n}$, the distribution of $Z_{n}$ depends on the distribution of $X_{n}$. Splett et al. [3], Poggiolini et al. [5], and Beygi et al. [6] have all derived models where $Z_{n}$ is a zero-mean, circularly symmetric sequence, whose power $P_{Z}=\mathbb{E}\left[\left|Z_{n}\right|^{2}\right]$ depends on the transmit power $P_{X}=\mathbb{E}\left[\left|X_{n}\right|^{2}\right]$ as

$$
P_{Z}=c_{0}+c_{3} P_{X}^{3}
$$

where $c_{0}$ and $c_{3}$ quantify the amplified spontaneous emission noise of the optical amplifiers and the $\mathrm{NLI}$, resp. The cubic relation (2) holds for both lumped and distributed amplification schemes, and it extends to multiple-wavelength $[3,5]$ and dualpolarization [5, 6] systems. For uncoded transmission with traditional modulation formats, the GN model has been shown to be very accurate in experiments and simulations.

Since $Z_{n}$ in (1) is additive and statistically independent of $X_{n}$, the channel capacity of the GN model (1)-(2) can be calculated exactly as [3, 7]

$$
C=\log _{2}\left(1+\frac{P_{X}}{P_{Z}}\right)
$$

using Shannon's well-known capacity expression [1, Sec. 24], [2, Ch. 9]. Considered as a function of the transmitted signal power $P_{X}$, the capacity has the peculiar behavior of reaching a peak and eventually decreasing to zero at high enough power, since the denominator of (3) increases faster than the numerator. The phenomenon, sometimes called the "nonlinear Shannon limit" in the optical communications community, seems to convey the message that reliable communication over nonlinear optical channels becomes impossible at high powers. In the last part of this paper, we question this pessimistic conclusion.

\section{Capacity and mutual information}

By Shannon's channel coding theorem, the channel capacity of a discrete-time memoryless channel, in bit/symbol, can be calculated as [1], [2, Ch. 7]

$$
C=\sup _{f_{X}} I(X ; Y),
$$

where $I(X ; Y)$ is the mutual information

$$
I(X ; Y)=\iint f_{X, Y}(x, y) \log _{2} \frac{f_{X, Y}(x, y)}{f_{X}(x) f_{Y}(y)} d x d y,
$$

$f_{X, Y}$ is the joint distribution of $X$ and $Y$, and $f_{X}$ and $f_{Y}$ are its respective marginal distributions. The capacity is often studied as a function of the transmitted 
signal power, $P_{X}=\mathbb{E}\left[|X|^{2}\right]$. The corresponding expression for channels with memory is, under certain assumptions on information stability [8],

$$
C=\lim _{n \rightarrow \infty} \sup _{f_{\boldsymbol{X}_{1}^{n}}} \frac{1}{n} I\left(\boldsymbol{X}_{1}^{n} ; \boldsymbol{Y}_{1}^{n}\right)
$$

where $\boldsymbol{X}_{i}^{j}=\left(X_{i}, X_{i+1}, \ldots, X_{j}\right)$ and $I\left(\boldsymbol{X}_{i}^{j} ; \boldsymbol{Y}_{i}^{j}\right)$ is defined as a multidimensional integral analogous with (4).

In this work, we are interested in channels with finite memory. Such channels have the property that the channel output $Y_{n}$ depends on a finite number $N$ of past input symbols, but not on the entire history, i.e.,

$$
f_{Y_{n} \mid \boldsymbol{X}_{1}^{n}}\left(y_{n} \mid \boldsymbol{x}_{1}^{n}\right)=f_{Y_{n} \mid \boldsymbol{X}_{n-N}^{n}}\left(y_{n} \mid \boldsymbol{x}_{n-N}^{n}\right),
$$

where $N$ is the channel memory. By expanding the right-hand side of (5) using the chain rule for mutual information [2, p. 24], it can be shown that for channels that satisfy (6) and any stochastic process $X_{n}$,

$$
C \geq I\left(X_{n} ; Y_{n} \mid \boldsymbol{X}_{n-N}^{n-1}\right),
$$

where $I\left(X_{n} ; Y_{n} \mid \boldsymbol{X}_{n-N}^{n-1}\right)$ is the conditional mutual information [2, p. 23].

\section{A finite-memory GN model}

Even highly dispersive optical fibers have a finite memory. The output does not depend what was transmitted into the fiber yesterday, or even a second ago. To account for this important feature, we propose a finite-memory GN model. The input-output relation is still given by (1), but the statistical transmit power $P_{X}$ in (2) is replaced with an empirical transmit power, which is a function of the actual transmitted sequence $X_{n}$ during a finite window of $N$ past input samples. Assuming a constant weight for all samples in this window, (2) is replaced by

$$
\begin{aligned}
& P_{Z}=c_{0}+c_{3} P_{n}^{3}, \\
& P_{n}=\frac{1}{N} \sum_{i=n-N}^{n-1}\left|X_{i}\right|^{2} .
\end{aligned}
$$

In the limit $N \rightarrow \infty$, the empirical power in (9) converges to the statistical power $P_{X}=\mathbb{E}\left[X_{n}\right]$, for any stationary, ergodic input sequence $X_{n}$. Hence, for large enough $N$, assuming uncoded transmission or coded schemes with short or moderate block lengths, the finite-memory GN model is equivalent to the regular GN model in the previous section, which can be regarded as an infinite-memory model.

For a suitable (large but finite) choice of $N$, the finite-memory model is more physically relevant than the traditional GN model, but much harder to analyze, since every output depends on a vector of inputs. A reasonable choice of $N$ is in the order of $N_{\text {sys }}=2 \pi\left|\beta_{2}\right| L R_{\mathrm{s}}^{2}$, where $\beta_{2}$ is the group velocity dispersion parameter, $L$ is the fiber length, and $R_{\mathrm{S}}$ is the symbol rate [9]. The finite-memory model is not accurate for small values of $N$, since the $\mathrm{GN}$ assumption relies on the central limit theorem $[5,6]$.

\section{Channel capacity results}

An exact expression for the channel capacity of the finite-memory GN model (1), (8)-(9) is unfortunately not available. Shannon's formula (3) does not apply, because the sequences $X_{n}$ and $Z_{n}$ are dependent. Furthermore, capacity estimation via (5) is numerically infeasible, since it would involve maximization over a high-dimensional space. We therefore resort to the lower bound (7), and furthermore constrain the input distribution to a ring constellation [4], with two discrete amplitudes $r_{1}, r_{2}$ and uniform phase. In this case, the right-hand side of (7) is

$$
\begin{aligned}
& I\left(X_{n} ; Y_{n} \mid \boldsymbol{X}_{n-N}^{n-1}\right) \\
& \quad=\sum_{s \in \mathcal{S}} \operatorname{Pr}\left\{P_{Z}=s\right\} \frac{2}{s} \int_{0}^{\infty} \rho b(g(\rho, s)) d \rho-\log _{2} e
\end{aligned}
$$

where $b(u)=-u \log _{2} u, \mathcal{S}$ is the set of $N+1$ possible values that $P_{Z}$ in (8)-(9) can take on when $\left|X_{i}\right| \in$ $\left\{r_{1}, r_{2}\right\}$,

$$
\begin{aligned}
g(\rho, s)= & \sum_{r \in\left\{r_{1}, r_{2}\right\}} \operatorname{Pr}\left\{\left|X_{n}\right|=r\right\} \\
& \cdot \exp \left(-\frac{\rho^{2}+r^{2}}{s}\right) I_{0}\left(\frac{2 \rho r}{s}\right),
\end{aligned}
$$

and $I_{k}(u)$ is the modified Bessel function of the first kind. The proof of (10) is omitted due to space constraints.

The radii and probabilities of the two rings are varied to maximize $I\left(X_{n} ; Y_{n} \mid \boldsymbol{X}_{n-N}^{n-1}\right)$ in (10), for given channel parameters $c_{0}, c_{3}$, and $N$ and a given transmit power $P_{X}$. In our numerical example, we use $c_{0}=3.27 \mu \mathrm{W}$ and $c_{3}=1.83 \mathrm{~mW}^{-2}$. The same values were used by Bosco et al. [7, 10], representing a standard single-mode fiber with length $L=8000 \mathrm{~km}$, parameters $\alpha=0.22 \mathrm{~dB} / \mathrm{km}, \beta_{2}=-21.7 \mathrm{ps}^{2} / \mathrm{km}$, and $\gamma=1.27(\mathrm{~W} \mathrm{~km})^{-1}$, ideal distributed amplification with $K_{T}=1$, WDM transmission with center frequency $\nu=190 \mathrm{THz}$ and bandwidth $B_{\mathrm{WDM}}=4 \mathrm{THz}$, and symbol rate $R_{\mathrm{s}}=32$ Gbaud.

The lower bound (10) is shown in Fig. 1 as a function of transmit power and channel memory $N$. We can see that as $N$ increases, the curves approach 


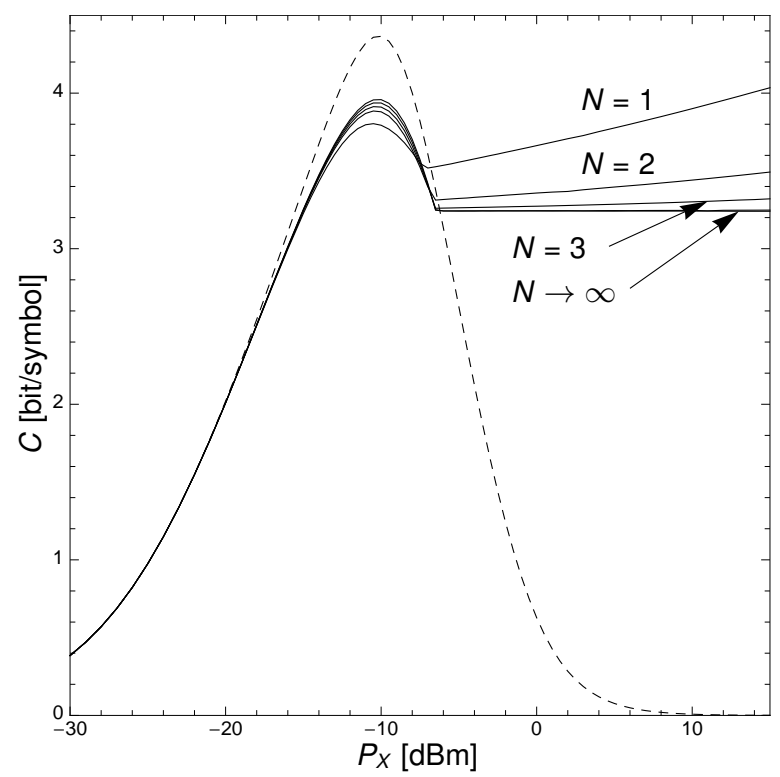

Fig. 1: Lower bounds (10) on the channel capacity $C$ vs. transmit power $P_{X}$ of the finite-memory GN model for varying channel memory $N$ (solid) and the exact capacity (3) of the regular (infinite-memory) GN model (dashed).

an asymptotic bound, marked $N \rightarrow \infty$. This asymptotic bound is plotted for $N=5$ and 10 (the two curves overlap), and it would look the same for, say, $N=N_{\text {sys }} \approx 1100$ or even higher. It has a peak at $3.96 \mathrm{bit} / \mathrm{symbol}$, after which it decreases to 3.24 bit/symbol.

The rudimentary lower bounds in Fig. 1 represent two-ring constellations only and can be improved by using other input distributions. For example, using so-called satellite constellations, a higher lower bound can be obtained that flattens out without decreasing [11, 12].

The exact channel capacity (3) of the infinitememory GN model (1)-(2) is included in Fig. 1 for reference. This is the $8000-\mathrm{km}$ curve shown in [10, Fig. 1(a)], rescaled by a factor of two to represent a single polarization. It is striking, and at first glance counterintuitive, that the channel capacity of the infinite-memory model is so different from the asymptotic capacity of the finite-memory model in the nonlinear regime. A mathematical explanation is that in general, $\lim _{N \rightarrow \infty} \sup _{f} C_{N}(f) \neq$ $\sup _{f} \lim _{N \rightarrow \infty} C_{N}(f)$. For a more intuitive explanation, we recall that a capacity-approaching transmission scheme should involve coding over a long block length, typically much longer than the channel memory $N$. Designing such long codes is possible, at least theoretically, for channel models with any finite memory, but not for infinite-memory models. Therefore, the two types of models have different channel capacities, not only for the example studied here but also for other nonlinear fiber channels, including dual polarization, wavelength multiplexing, multimode fibers, etc.

\section{Conclusions}

We extended the popular GN model for nonlinear fiber channels with a parameter to account for the channel memory. The new channel model is given by (1) and (8)-(9). For any finite memory, its channel capacity is quite different from that of the regular (infinite-memory) GN model in the nonlinear regime. Hence, infinite-memory channel models, although accurate for uncoded transmission, should not be used in capacity analysis. Their capacities do not bound the achievable rates of the underlying physical links.

We intentionally avoid using the concept "nonlinear Shannon limit." It is an artifact of the use of infinite-memory channel models and has no known analogy for finite-memory models, which are more physically meaningful. The real fiber link does not suffer from the same vanishing capacity at high transmit powers as the regular GN model and other infinite-memory models do. The true capacity for nonlinear, dispersive channels remains an open problem.

\section{Acknowledgments}

The author wishes to thank Magnus Karlsson and other members of the FORCE center at Chalmers for many helpful discussions. Funding from SSF and VR is gratefully acknowledged.

\section{References}

1 C. E. Shannon, Bell Syst. Tech. J., 27, 379, 623 (1948).

2 T. M. Cover et al., Elements of Information Theory, 2nd ed., Wiley (2006).

3 A. Splett et al., Proc. ECOC, 41 (1993).

4 R.-J. Essiambre et al., J. Lightw. Technol., 28, 662 (2010).

5 P. Poggiolini et al., IEEE Photon. Technol. Lett., 23, 742 (2011).

6 L. Beygi et al., IEEE Trans. Commun., 60, 3440 (2012).

7 G. Bosco et al., Optics Express, 19, B440 (2011).

8 S. Verdú et al., IEEE Trans. Inf. Theory, 40, 1147 (1994).

9 E. Ip et al., J. Lightw. Technol., 25, 2033 (2007).

10 G. Bosco et al., Optics Express, 20, 19610 (2012).

11 E. Agrell, http://arxiv.org/abs/1209.2820, (2012).

12 E. Agrell et al., Proc. IPC, 316 (2012). 